\title{
CALIDAD DE VIDA DEL PROFESIONAL DE ENFERMERÍA EN DOS UNIDADES DE CUIDADO INTENSIVO DE BOGOTA DC
}

Sandra Forero, Claudia Naranjo*

\section{Resumen}

Esta investigación describe y analiza los factores laborales (remuneración económica, carga de trabajo laboral, horas de descanso) que influyen en la calidad de vida de los profesionales de enfermería en dos unidades de cuidado intensivo (UCI) de Bogotá. De igual forma aporta elementos para enriquecer en el futuro el desarrollo de nuevos estudios, leyes o políticas de salud, que no solo beneficien al enfermero, sino a través de él, al paciente en condiciones criticas. La metodología utilizada es cualicuantitativa con un diseño descriptivo transversal. La muestra fue de 16 profesionales de enfermería de dos UCI en Bogotá, que accedieron participar en el estudio luego de evaluar las condiciones y objetivos del mismo. Como antecedentes y referencia del trabajo se tuvieron en cuenta las publicaciones sobre calidad de vida de médicos y odontólogos generales en Santafé de Bogotá 1998-1999 y calidad de vida del profesional de enfermería que labora en las unidades renales de Bogotá DC 2001. Los resultados mostraron una población de sexo femenino, en su mayoría jóvenes,solteras, con contratación por prestación de servicios, cuya remuneración oscila entre uno y dos millones de pesos. El tiempo de descanso de este personal varía entre cuatro y cinco horas, lo cual en criterio de las encuestadas no es suficiente. Las causas de morbilidad más frecuentes fueron en su orden: lesiones osteomusculares, enfermedad ácido-péptica, migraña, irritabilidad o cambios de carácter, Con respecto a las preguntas relacionadas sobre las actividades desarrolladas por estas profesionales durante su tiempo de descanso, la mayoría respondió realizar aquellas de poca actividad física y que pueda compartir con su familia.

\section{Introducción}

El compromiso social, científico, pero sobre todo humano que el profesional de enfermería adquiere con su comunidad, está estrechamente ligado con su capacidad de identificar cada uno de los factores que contribuirán en forma progresiva al mejoramiento del cuadro clínico del paciente. Esto significa coordinar en forma efectiva el conocimiento técnico y científico, en especial al ofrecer cuidado al paciente críticamente enfermo. Es importante destacar la complejidad de las salas de cuidado intensivo, las exigencias y el estrés al que a diario se ven sometidos los profesionales que laboran en ellas, donde lo más importante es brindar en forma adecuada y ética el cuidado, que es el principal quehacer de la enfermería. Se resalta que este compromiso exige cuidar y reconocer primero en sí mismo las condiciones de

Enfermeras estudiantes de especialización en cuidado intensivo. calidad de vida adecuadas, para que se puedan proyectar a los pacientes.

El tema de la calidad de vida de los profesionales de enfermería que laboran en la UCI fue generado por el interés de las autoras de contribuir en la identificación y resolución de un problema que enmarcado en el actual escenario nacional, de violación de los derechos humanos y de incertidumbre social, constituye un elemento esencial para desarrollar con eficacia el papel que socialmente estamos llamados a desempeñar como ciudadanos. El profesional de enfermería ejerce sobre el paciente y la familia un papel importante, por lo tanto su calidad de vida (descanso, estado de ánimo, remuneración, etc.) repercute en forma directa en el cuidado que brinda a sus pacientes.

Por último es importante identificar las condiciones adecuadas de calidad de vida para los profesionales 
de enfermería, para que sirvan de retroalimentación grupal e individual, de manera que permita elaborar estrategias para favorecer los elementos protectores a nivel laboral que promuevan el desarrollo integral de estos profesionales.

\section{El profesional de enfermería en la UCI}

La UCI se caracteriza por ser el lugar dentro de una institución prestadora de atención donde se hospitalizan los pacientes críticos y por esto deben estar dotadas de personal bien entrenado y de alta tecnología para la vigilancia permanente. La práctica de enfermería abre un campo bastante específico con la asignación de una serie de funciones dependientes, interdependientes e independientes, las cuales permiten al enfermero desarrollar sus cualidades y talentos. La competencia técnica del enfermero en la UCI debe estar fundamentada en una actividad moral, ya que la deshumanización no proviene de la ciencia y la tecnología en sí misma, sino de un mal uso, por parte de la persona que la practica. Los conocimientos básicos del enfermero deben abarcar también los fundamentos de resolución de problemas, análisis y toma de decisiones. Debe ser capaz de analizar los datos de valoración, relacionar aquellos que tengan algo en común, deducir conclusiones válidas y hacer juicios de valor que contribuyan a la mejoría del paciente. Es por tanto, un pilar fundamental en la atención que se brinda al paciente en estado crítico, es quien con sus cuidados y procedimientos oportunos hace que la recuperación del paciente sea lo más exitosa posible.

\section{Metodología}

Este es un estudio de observación descriptivo de tipo transversal, ya que muestra los datos específicos en un tiempo y espacio determinados, que en este caso son dos UCI de Bogotá. La muestra está comprendida por 16 profesionales de enfermería de diferentes turnos, a quienes se les aplicó una entrevista estructurada por las investigadoras dirigida a identificar los indicadores que pueden influir en la calidad de vida de los enfermeros.

La descripción permitirá establecer las características que van a determinar si los indicadores influyen o no en la calidad de vida de los enfermeros que trabajan en las dos UCI de Bogotá.

Los criterios de inclusión fueron establecidos por las autoras las cuales determinaron que los sujetos de la investigación fueran todos los profesionales de enfermería que trabajen en UCI sin importar el turno, el tiempo que lleven allí y si son o no especialistas en cuidado intensivo.

\section{Resultados}

- Se determinó a través del estudio que el personal de enfermería que laborar en dos UCI en Bogotá, son de sexo femenino, poseen un rango de edad entre 20 -30 años, lo cual nos permite concluir que a pesar de las actuales condiciones sociales y culturales en las que el género no es un condicionamiento en la escogencia profesional, el cuidado de enfermería sigue siendo tradicionalmente dado por mujeres.

- Se determinó con el presente trabajo, que las enfermeras que laboran en las unidades de cuidado intensivo del estudio, reciben una remuneración por su trabajo de 1.5 millones a 2 millones de pesos y que su nivel de egresos es igual, asociado con el hecho de que este personal es contratado por las entidades en la modalidad de prestación de servicios, en la que el profesional debe asumir los costos de ARP, EPS y en la mayoría de los casos pólizas de cumplimiento que exigen las instituciones. Esta situación nos permite concluir que no existe dentro de este grupo la posibilidad de realizar un ahorro constante.

- Se encontró que las enfermeras del estudio se exponen a situaciones que causan estrés. Las más importantes fueron no disponer del tiempo suficiente para desarrollar todas las actividades y no tener los elementos necesarios, para los procedimientos, lo cual demuestra el gran interés del personal por realizar sus actividades con un buen nivel de calidad.

- Se halló que las enfermeras descansan entre 4-5 horas y para que este descanso sea efectivo creen necesitar 8 a 10 horas de reposo diarias, pues está relacionado con los cambios de humor. Esto nos demuestra que el personal de enfermería no posee suficiente tiempo de 
descanso, lo cual no solo genera irritabilidad, sino que puede afectar en el futuro su desempeño laboral y social. La prevalencia de enfermedades laborales entre el personal de enfermería fueron las relacionadas con dolores musculares en mayor frecuencia migraña y gastritis en porcentajes también representativos para el estudio, lo cual demuestra que la complejidad del servicio, las características críticas del paciente y la falta de elementos adecuados para la realización de las actividades, generan en el personal de enfermería que labora en la UCI, la aparición o exacerbación de esta clase de afecciones.

\section{Conclusiones $y$ recomendaciones}

- Los factores laborales más asociados a la calidad de vida de las enfermeras que laboran en las unidades de cuidado intensivo de la Clínica Fundadores y del Hospital Central de la Policía, son la carga laboral, la remuneración económica, las horas de descanso y el tipo de contratación.

- El factor relacionado con el numero de horas de descanso, está estrechamente ligado con los cambios de carácter, con la aparición y exacerbación de enfermedades como migraña, que a su vez tienen influencia en su ambiente familiar y social.

- Las enfermedades más frecuentes del personal que labora en las dos UCI, son de origen gastrointestinal y las osteomusculares.

Se recomienda:

- Estimular la asistencia del personal que trabaja en la UCI a cursos de actualización científicos y técnicos, así como actividades de enriquecimiento y mejoramiento personal, como las recreativas y las técnicas de relajación.
- Fomentar la participación del profesional de enfermería que labora en la UCI, en los procesos de cambio y mejoramiento de estos servicios, estimulando la comunicación y liderazgo.

- Proporcionar al profesional de enfermería que labora en la UCI, estímulos laborales que se reflejen en su calidad de vida.

- Es necesario ampliar el estudio con una población mayor, para tener mejor perspectiva de la calidad de vida actual del enfermero que labora en la UCI en Colombia.

\section{Lecturas recomendadas}

- Barrera, M M y otros. Calidad de vida de las enfermeras que laboran en las unidades renales de Bogotá, 2000-2001. Bogotá: FUCS. Tesis de postgrado. Facultad de Enfermería. Fundación Universitaria Ciencias de la Salud.

- Buitrago, R. Acta colombiana de Cuidado Intensivo. Vol. 4, No.2. Julio 2001.

- Colell, R. Atención al enfermo terminal. En: Revista ROL de Enfermería mayo 1993; 177.

- Creus, M. Calidad de vida enfermera. En: Revista ROL de Enfermería. 154. abril 1992; 154 Delar BB Fisiología médica. 2a. edición Barcelona Mayson 1999.

- Delar BB.Medicina del dolor y cuidados paliativos. Cali: Universidad libre de Cali 1998.

- Gómez ME y otros. Fundamentos de Medicina, el paciente en estado critico. $2^{a}$ edición. Corporación para Investigaciones Biológicas. Medellín Colombia. CIB.1997.

- Kozier B.y otros. Conceptos y temas de la práctica de Enfermería. Interamericana: 1995.

- Llopis S y otros. Motivación laboral, creación de círculos de calidad. En: Revista ROL de Enfermería. Abril 1993:176.

- Millán CP y otros. Calidad de vida de médicos y odontólogos generales en Santa Fe de Bogotá 1998-1999. Bogotá: FUCS. Tesis postgrado. Gerencia en Salud, Fundación Universitaria Ciencias de la Salud.

- Vásquez T y otras. Investigación en enfermería, programa de magíster a distancia en enfermería. Cali: Universidad Libre de Cali: Facultad de salud departamento de Enfermería 1993.

- Velandia AL Historia de la Enfermería en Colombia, Bogotá: Universidad Nacional de Colombia, 1995.

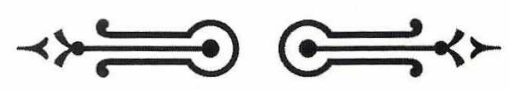

